\title{
Neonatal abstinence syndrome due to prenatally citalopram exposure: A case report
}

Sara Erol, M.D. ${ }^{a}$, Beyza Ozcan, M.D. ${ }^{a}$, Istemi H. Celik, M.D., Assoc. Prof. ${ }^{a}$, Ahmet Y. Bas, M.D., Assoc. Prof. ${ }^{a}$ and Nihal Demirel, M.D., Assoc. Prof. ${ }^{a}$

\begin{abstract}
Neonatal abstinence syndrome (NAS) due to prenatally exposure to citalopram can develop during the first days of life even with low dose of drug exposure. Supportive management is the first choice but phenobarbital can be used in treatment of this syndrome. Breastfeeding should not be interrupted. These neonates should be followed both for NAS and neurodevelopmental outcome. In this article, we reported a newborn with NAS due to citalopram exposure with a lower dose than previously reported in the literature, during the last six months of pregnancy. Phenobarbital was used because of non-pharmacological treatment failure.

Key words: neonatal abstinence syndrome, citalopram, Phenobarbital.
\end{abstract}

http: / / dx.doi.org/10.5546/ aap.2017.eng.e424

To cite: Erol S, Ozcan B, Celik IH, et al. Neonatal abstinence syndrome due to prenatally citalopram exposure: A case report. Arch Argent Pediatr 2017;115(6):e424-e427.

\section{INTRODUCTION}

Psychiatric disorders such as major depression, anxiety, and obsessive compulsive disorders are common during and after pregnancy. A large study from United States showed a prevalence of $13 \%$ for mood and anxiety disorders in pregnant or postpartum women. ${ }^{1}$ Antidepressants are commonly used to treat these disorders. Adequate treatment of depression during pregnancy is of great importance for maternal, fetal and neonatal health. ${ }^{2}$ Selective serotonin reuptake inhibitors (SSRIs) are the most prescribed group of antidepressants, also in pregnant women. ${ }^{3}$

a. Neonatology, Etlik Zubey de Hanim Women's Health Teaching and Research Hospital, Ankara, Turkey.

E-mail address:

Sara Erol, M.D.: sarasurmeli@gmail.com

Funding: None.

Conflict of interest: None.

Received: 12-27-2016

Accepted: 7-3-2017
SSRIs, especially paroxetine, fluoxetine and sertraline, during late pregnancy may lead to neonatal abstinence syndrome (NAS). This syndrome is also called as poor neonatal adaptation. ${ }^{4}$ It includes symptoms such as jitteriness, convulsion, abnormal crying, feeding problems, respiratory distress and hypoglycemia. ${ }^{5}$ We reported a newborn with NAS due to citalopram exposure during the last six months of pregnancy.

\section{Case report}

A $3010 \mathrm{~g}$ male infant, length $50 \mathrm{~cm}$ and head circumference $33 \mathrm{~cm}$, was delivered by cesarean section at 36 weeks of gestation. The mother was 40 year old and had good prenatal care and history of major depression. She had been taking citalopram $10 \mathrm{mg} /$ day beyond the last two trimesters of her pregnancy. At birth, the infant had Apgar scores of 8, 9 and 10 at 1, 5 and 10 minutes. The patient was admitted to the neonatal intensive care unit because of respiratory distress in the first hour after birth. On admission, he was tachypneic with a respiratory rate of 68 per minute with intercostal and subcostal retractions. Complete blood count, arterial blood gases, blood glucose level and serum electrolytes including calcium and magnesium, and chest $\mathrm{X}$-ray were in normal ranges. Serum C-reactive protein (CRP) was negative. Respiratory distress was treated with supplemental oxygen and gradually improved during the first day.

On postnatal day 3, jitteriness and hyperirritability occurred. Hypoglycemia $(25 \mathrm{mg} / \mathrm{dl})$ and hypocalcaemia (ionized calcium: $3.1 \mathrm{mg} / \mathrm{dl}$ ) were diagnosed. Jitteriness and hyperirritability continued despite intravenous glucose and calcium replacement treatment. Muscle tone increased within hours and opisthotonic posture began to appear intermittently. He had hyperreactive deep tendon and exaggerated Moro reflexes. The modified Finnegan score was performed for NAS. The result was found as 8 , corresponding to severe NAS. Infant was subsequently scored at least every 2 hours by the neonatologist. Non- 
pharmacological treatments such as minimizing environmental stimuli, adequate rest and sleep, breastfeeding and swaddling were started. Phenobarbital as $5 \mathrm{mg} / \mathrm{kg} /$ day divided every 12 hours was started because 3 consecutive scores were $>8$ despite non-pharmacological treatments. Cranial USG and EEG were performed for differential diagnosis with normal results. After three days of phenobarbital treatment, jitteriness, muscle rigidity and hyperirritability reduced; the modified Finnegan score was $<8$ and phenobarbital dose was gradually decreased and stopped on postnatal day 14 . The clinical signs gradually improved at postnatal day 10 . The patient was discharged with normal physical examination on day 16.

\section{DISCUSSION}

Placental transfer of SSRIs has been demonstrated experimentally both in animals and in humans. These drugs had not placental metabolism. Placental drug transfer is also determined by duration of drug exposure, liposolubility, protein binding, volume of distribution and other pharmacokinetic factors. ${ }^{6}$ By definition, antidepressant abstinence or discontinuation syndrome occurs in the first few days after abrupt drug cessation, as after delivery, when the drug concentrations from the newborn's serum are not detectable or too low to have any biological effect; most common reported symptoms include irritability, sleep disturbances, abnormal crying, rigidity and tremor. ${ }^{7}$ Diagnosis is usually performed by prenatal history, clinical findings and Finnegan score. Finnegan score was originally developed to diagnose neonatal opioid abstinence ${ }^{8}$ but has also been used to assess neonatal symptoms in SSRI exposed infants. ${ }^{9}$

The recent study showed that most of infants born to mothers with SSRI treatment during pregnancy were healthy in the neonatal period. Severe abstinence syndrome was reported in $3-13 \%$ in these studies whereas mild abstinence rate was $22 \%$ due to maternal SSRIs exposure. The symptoms mainly arise from the central nervous system. Hypoglycemia and respiratory symptoms were found commonly..$^{10}$ Neonatal abstinence syndrome due to SSRIs had been reported in 93 cases including 6 cases with citalopram by 2003. ${ }^{11}$ A more recent retrospective cohort study by Forsberg et al. evaluated 71 newborns exposed to citalopram during the third trimester with a median dosage $20 \mathrm{mg} /$ day $\left(5-80 \mathrm{mg} /\right.$ day).${ }^{10}$ Severe (Finnegan score $\geq 8$ ) and mild (Finnegan score 4-7) abstinence treated with non-pharmacological therapy were diagnosed in 2 and 18 newborns, respectively. Respiratory distress and hypoglycemia were found in 5 and 8 newborns whereas 10 of the newborns were premature. They did not find any correlation between SSRIs and respiratory distress. Nordeng et al. reported 5 infants with NAS due to SSRIs including 1 with citalopram. ${ }^{12}$ He was a male infant exposed to citalopram with the dosage of $20 \mathrm{mg}$ / day starting from 5th month of pregnancy and increased to $30 \mathrm{mg}$ / day 2 months before delivery. He did not need medical treatment (Finnegan scores 4-6) and was discharged at the postnatal 7th day. There is no safe citalopram dose to prevent NAS. A lower dose exposure than previous studies such as our patient, $10 \mathrm{mg} / \mathrm{kg}$, may lead to NAS. In our patient, respiratory distress which was usually companied with SSRIs use in pregnancy resolved in $24 \mathrm{~h}$. Hypoglycemia and hypocalcaemia responded intravenous glucose and calcium as reported in previous studies. Since our patient was a premature, the reason for hypoglycemia and hypocalcaemia could be prematurity not NAS. But, neuromuscular findings were resolved after phenobarbital treatment.

Serum levels of citalopram in infants were reported to be $0.9 \%-4.3 \%$ of the mothers' serum levels 2 weeks to 2 months after delivery. Infant citalopram intake dose by breastfeeding was reported to be $0.7 \%-9 \%$ of maternal dose after adjusting weight and considering citalopram bioavailabilitiy as $100 \% .{ }^{13}$ Breastfeeding should be offered in these patients because these studies did not reveal citalopram-associated effects in infants during breastfeeding whereas formula feeding of these infants was found to be a risk factor for NAS. ${ }^{4}$ Therapeutic drug monitoring by measuring citalopram concentrations in serum of both mother and newborn may prevent drugassociated side effects. With today's extremely short duration of hospital stay for newborns and the longer half-life of SSRIs, the symptoms may even occur after discharge from hospital. ${ }^{10}$ In our center, the infants exposed to antidepressants stayed in hospital at least 72-96 hours.

Studies on long term effects of prenatal SSRIs are limited. Maternal depression is a major risk factor for neurodevelopmental problems. ${ }^{14}$ There seem to be no major effects, but further studies are needed.

Management of the neonate includes both pharmacological and non-pharmacological care. Non-pharmacological therapy is the first option 
in all cases, and may be sufficient in cases of mild abstinence. Non-pharmacological therapy is easily acceptable, less expensive, and less controversial. Non-pharmacological therapy can be attempted in all infants before initiating pharmacological therapy. Successful management comprises gentle handling, demand feeding, and careful avoidance of waking the sleeping infant. Continuous minimal stimulation practices with dim light and low noise must be implemented in all neonates. Frequent feeds, high calorie formula, and thickened feeds may meet nutritional and metabolic demands. ${ }^{15}$ Phenobarbital is a drug of choice for non- opiate NAS. Phenobarbital does not prevent seizures at the dosage administered for abstinence, nor improves gastrointestinal symptoms. ${ }^{15}$ We observed that neurological symptoms were improved in our patient.

In conclusion, neonatal maladaptation and NAS due to prenatally SSRIs' exposure such as citalopram can develop in first days of life even with low dose of drug exposure. Supportive management is the first choice but phenobarbital can be used in treatment of this syndrome. Breastfeeding should be continued preferably. These neonates should be followed both for NAS and neurodevelopmental outcome.

\section{REFERENCES}

1. Vesga-Lopez O, Blanco C, Keyes $\mathrm{K}$, et al. Psychiatric disorders in pregnant and postpartum women in the United States. Arch Gen Psychiatry 2008;65(7):805-15.

2. Patil AS, KullerJA, RheeEH. Antidepressants in pregnancy: a review of commonly prescribed medications. Obstet Gynecol Surv 2011;66(12):777-87.

3. Stephansson O, Granath F, Svensson T, et al. Drug use during pregnancy in Sweden - assessed by the Prescribed
Drug Register and the Medical Birth Register. Clin Epidemiol 2011;3:43-50.

4. Kieviet N, van Ravenhorst M, Dolman KM, et al. Adapted Finnegan scoring list for observation of antidepressant exposed infants. J Matern Fetal Neonatal Med 2015;28(17):2010-4.

5. Reis M, Källén B. Delivery outcome after maternal use of antidepressant drugs in pregnancy: an update using Swedish data. Psychol Med 2010;40(10):1723-33.

6. Klinger G, MerlobP.SelectiveSerotonin Reuptake Inhibitor Induced Neonatal Abstinence Syndrome. Isr J Psychiatry Relat Sci 2008;45(2):107-13.

7. Koren G, Matsui D, Einarson A, et al. Is maternal use of selective serotonin reuptake inhibitors in the third trimester of pregnancy harmful to neonates? CMAJ 2005; 172(11):1457-9.

8. Finnegan LP, Connaughton JF, Kron RE Jr, et al. Neonatal abstinence syndrome: assessment and management. Addict Dis 1975;2(1-2):141-58.

9. Levinson-Castiel R, Merlob P, Linder N, et al. Neonatal abstinence syndrome after in utero exposure to selective serotonin reuptake inhibitors in term infants. Arch Pediatr Adolesc Med 2006;160(2):173-6.

10. Forsberg L, Navér L, Gustafsson LL, et al. Neonatal AdaptationinInfantsPrenatallyExposed toAntidepressantsClinical Monitoring Using Neonatal AbstinenceScore.PLoS One 2014;9(11):e111327.

11. Sanz EJ, De-las-Cuevas C, Kiuru A, et al. Selective serotonin reuptake inhibitors in pregnant women and neonatal withdrawal syndrome: a database analysis. Lancet 2005;365(9458):482-7.

12. Nordeng H, Lindemann R, Perminov KV, et al. Neonatal withdrawal syndrome after in utero exposure to selective serotonin reuptake inhibitors. Acta Paediatr 2001;90(3): 288-91.

13. Franssen EJ, Meijs V, Ettaher F, et al. Citalopram serum and milk levels in mother and infant during lactation. Ther Drug Monit 2006;28(1):2-4.

14. Nulman I, Koren G, Rovet J, et al. Neurodevelopment of children following prenatal exposure to venlafaxine, selective serotonin reuptake inhibitors, or untreated maternal depression. Am J Psychiatry 2012;169(11):1165-74.

15. Kocherlakota P. Neonatal abstinence syndrome. Pediatrics 2014;134(2):e547-61. 